\title{
Magneto-Optical Effect of One-Dimentional Magnetophotonic Crystal Utilizing the Second Photonic Band Gap
}

\author{
H. Uchida ${ }^{1 *}$, K. Tanizaki ${ }^{1}$, A. B. Khanikaev' ${ }^{1}$ A. A. Fedyanin ${ }^{2}$, P. B. Lim ${ }^{1}$, and M. Inoue ${ }^{1,3}$ \\ ${ }^{1}$ Department of Electrical and Electronic Engineering, Toyohashi University of Technology, \\ 1-1 Tempaku, Toyohashi, Aichi 441-8580, Japan \\ ${ }^{2}$ Department of Physics, Moscow State University, Moscow 119992, Russia \\ ${ }^{3}$ JST-CREST, 4-1-8, Honcho, Kawaguchi, Saitama 332-0012, Japan
}

(Received 11 September 2006)

\begin{abstract}
We fabricated new one-dimensional magnetophotonic crystal (1D-MPC) utilizing the second and third photonic band gaps where localized modes existed. Structure of the 1D-MPC was $\left(\mathrm{Ta}_{2} \mathrm{O}_{5} / \mathrm{SiO}_{2}\right)^{5} / \mathrm{Bi}: \mathrm{YIG} /\left(\mathrm{SiO}_{2} / \mathrm{Ta}_{2} \mathrm{O}_{5}\right)^{5}$ with optical thicknesses of $3 \lambda / 4$ for $\mathrm{Ta}_{2} \mathrm{O}_{5}$ and $\mathrm{SiO}_{2}$ dielectric layers and $\lambda / 2$ for $\mathrm{Bi}$ :YIG defect layer, where $\lambda$ is a wavelength of a localized mode in the second photonic band gap. Faraday rotation at the localized mode in the second photonic band gap was enhanced, which was confirmed by calculation using $4 \times 4$ matrix method.
\end{abstract}

Key words : magnetophotonic crystal, MPC, second photonic band gap, Bi:YIG, Faraday rotation

\section{Introduction}

In recent years, one-dimensional magnetophotonic crystals (1D-MPCs) have attracted much attention due to their unique properties, such as control of propagation of light by applying magnetic field, enhancement of Faraday rotation and high transmittance at a localized mode in the photonic band gap (PBG) [1]. The 1D-MPCs consist of a transparent magnetic film as a defect layer and periodic dielectric films as Bragg mirrors. Linear and nonlinear magneto-optical (MO) properties of the1D-MPCs were investigated for the first PBG [2-5]. However, up to date, high order PBGs of the 1D-MPCs have not been discussed yet.

In this article, we investigated the second and the third PBGs of the 1D-MPC to clarify their optical and magneto-optical properties. In the 1D-MPC, high order PBGs appear in shorter wavelength distance than one between the first and the second PBGs. Adjacent multiple PBGs of the 1D-MPC will be used for applicative devices, such as a photonic band pass filter and so on. Goal of our study is to obtain not only the 1D-MPC but also two- and threedimensional (2D- and 3D-) MPCs. Idea of utilizing high order MPC may be one of solutions for difficulty of

*Corresponding author: Tel: +82-532-44-6731,

Fax:+82-532-44-6757, e-mail: uchida@eee.tut.ac.jp fabrication of 2D- and 3D-MPCs using magneto-optical material like magnetic garnet.

\section{Experimental}

Structure of the 1D-MPC is $\left(\mathrm{Ta}_{2} \mathrm{O}_{5} / \mathrm{SiO}_{2}\right)^{5} / \mathrm{Bi}: \mathrm{YIG} /$ $\left(\mathrm{SiO}_{2} / \mathrm{Ta}_{2} \mathrm{O}_{5}\right)^{5}$ formed on a fused quartz substrate as shown in Fig. 1. Wavelength $\lambda$ at a localized mode in the second PBG was designed to be $900 \mathrm{~nm}$. Optical length of $\mathrm{Ta}_{2} \mathrm{O}_{5}$ and $\mathrm{SiO}_{2}$ was $3 \lambda / 4$; that of $\mathrm{Bi}: \mathrm{YIG}$ was $\lambda / 2$. Since refractive index $\mathrm{n}_{\mathrm{Ta} 2} \mathrm{O}_{5}$ is 2.05 , thickness $\mathrm{d}_{\mathrm{Ta}_{2} \mathrm{O} 5}$ is $329 \mathrm{~nm}$; $\mathrm{n}_{\mathrm{SiO}_{2}}=1.45, \mathrm{~d}_{\mathrm{SiO}_{2}}=466 \mathrm{~nm} ; \mathrm{n}_{\mathrm{Bi}: \mathrm{YIG}}=2.36, \mathrm{~d}_{\mathrm{Bi}: \mathrm{YIG}}=191 \mathrm{~nm}$.

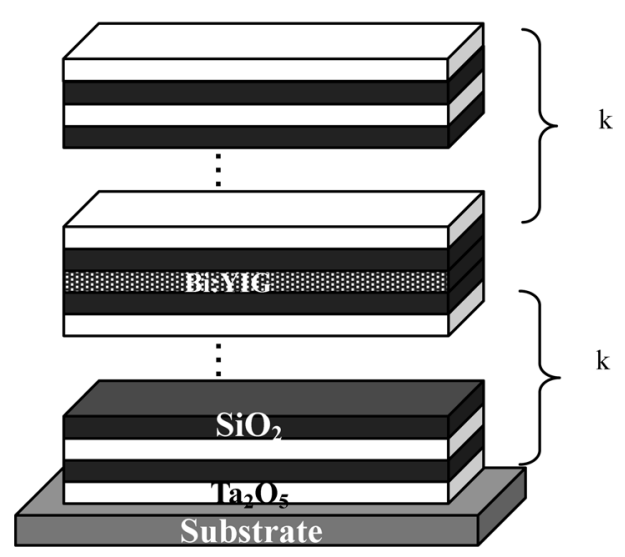

Fig. 1. Structure of 1D-MPC. 
Table 1. Conditions of deposition by RF magnetron sputtering method

\begin{tabular}{lccc}
\hline \hline & $\mathrm{Ta}_{2} \mathrm{O}_{5}$ & $\mathrm{SiO}_{2}$ & $\mathrm{Bi}: Y I G$ \\
\hline Sputtering gas & $\mathrm{Ar}: 8 \mathrm{ccm}$ & & $\mathrm{Ar} 6.3 \mathrm{ccm}$ \\
& $\mathrm{O}_{2}: 2 \mathrm{ccm}$ & & \\
Pressure & $10 \mathrm{mTorr}$ & & $3 \mathrm{mTorr}$ \\
Substrate temperature & 100 degrees & & rees \\
Sputtering power & $150 \mathrm{~W}$ & $100 \mathrm{~W}$ & $100 \mathrm{~W}$ \\
\hline
\end{tabular}

First, five pairs of the dielectric layers of $\mathrm{Ta}_{2} \mathrm{O}_{5}$ and $\mathrm{SiO}_{2}$, the first Bragg reflector, were deposited on the fused quartz substrate by using RF magnetron sputtering method, whose fabrication conditions of films are shown in Table 1. On the dielectric layers, a Bi:YIG defect layer was also deposited by RF magnetron sputtering method; it was heated in air at $700{ }^{\circ} \mathrm{C}$ for 15 minutes for crystallization of the magnetic garnet. Five pairs of dielectric films, the second Bragg reflector, were subsequently deposited. Finally, the 1D-MPC utilizing the second PBG around $900 \mathrm{~nm}$ was fabricated.

A field-emission scanning electron microscope (FESEM, JSM-6700F, JEOL) was used for observation of the fabricated 1D-MPC. Transmissivity of the 1D-MPC was measured by an ultraviolet-visible-infrared spectrophotometer (UV-3150, Shimadzu). Magnetic properties were measured by a vibration sample magnetometer (VSM, TM-VSM261483HGC, Tamagawa). A homemade magnetooptical measurement system was used for measurements of Faraday rotation angles.

\section{Calculation Method}

To simulate optical and magneto-optical properties we used $4 \times 4$ matrix method of Ref. [1]. This method is generalization of the transfer matrix method [2] on multilayered structures where at least one of the layers represents magneto-optical material. Following Ref. [1], we introduce transfer matrix $\hat{\Phi}_{n}$, which matches amplitudes of electric and magnetic fields of the EM wave at the boundaries of the n-th layer:

$$
\left[\begin{array}{c}
E_{x} \\
E_{y} \\
H_{x} \\
H_{y}
\end{array}\right]^{n}=\hat{\boldsymbol{\Phi}}_{n}\left[\begin{array}{l}
E_{x} \\
E_{y} \\
H_{x} \\
H_{y}
\end{array}\right]^{n-1}
$$

where $\mathrm{E}_{\mathrm{x}}\left(\mathrm{E}_{\mathrm{y}}\right)$ and $\mathrm{H}_{\mathrm{x}}\left(\mathrm{H}_{\mathrm{y}}\right)$ are amplitudes of the $x-(y-)$ projection of electric and magnetic fields, respectively, and EM wave propagates along $z$-direction, which coin- cides with the axis of the layered structure.

In the calculation, structure contains the pair of identical Bragg reflectors of $\mathrm{Ta}_{2} \mathrm{O}_{5}$ and $\mathrm{SiO}_{2}$ layers and one defect layer of Bi:YIG. The total transfer matrix for the 1D-MPC, which relates amplitudes at the last boundary of the last layer to amplitudes at the first boundary of the first layer, and therefore completely describes optical and magneto-optical properties of the structure, can be written in the following form:

$$
\hat{\Phi}=\left(\hat{\Phi}^{\mathrm{Ta}_{2} \mathrm{O}_{5}} \hat{\Phi}^{\mathrm{SiO}_{2}}\right)^{N} \hat{\Phi}^{\mathrm{Bi}: \mathrm{YIG}}\left(\hat{\Phi}^{\mathrm{SiO}_{2}} \hat{\Phi}^{\mathrm{Ta}_{2} \mathrm{O}_{5}}\right)^{N}
$$

where $\mathrm{N}$ is the repetition number of the $\mathrm{Ta}_{2} \mathrm{O}_{5}-\mathrm{SiO}_{2}$ pairs. When amplitudes of the fields are determined from equations (1) and (2), the well-known equation for the Faraday rotation angle can be used as

$$
\theta_{F}=\frac{1}{2} \arctan \left(\frac{2 \operatorname{Re}(\chi)}{1-|\chi|^{2}}\right)
$$

where $\chi$ is the ratio of the $y$ and $x$ components of the electric or magnetic field at the fixed polarization of the incident wave.

\section{Results and Discussion}

Fig. 2 shows a cross-section SEM image of the fabricated 1D-MPC having a localized mode inside the second PBG around $900 \mathrm{~nm}$. Film thicknesses measured by FESEM were $307 \mathrm{~nm}$ for $\mathrm{Ta}_{2} \mathrm{O}_{5}$ and $454 \mathrm{~nm}$ for $\mathrm{SiO}_{2}$ on averages, and $260 \mathrm{~nm}$ for Bi:YIG. Because our RF magnetron sputtering system does not have monitoring system of film thickness during deposition, each film thickness of the 1D-MPC is not exactly equal to designed one. In the fabricated 1D-MPC, the saturation magnetization was about $1.3 \mathrm{kG}$ as shown in Fig. 3; the saturation magnetic

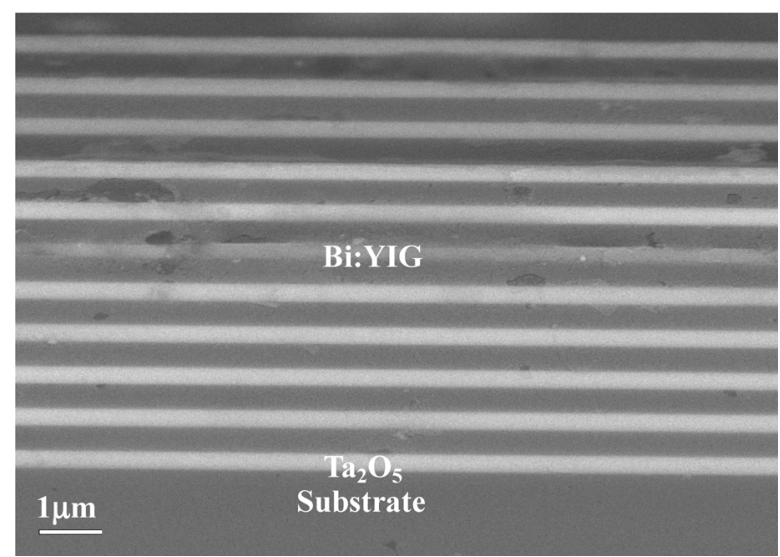

Fig. 2. Cross-section SEM image of the 1D-MPC having a localized mode in the second PBG around $900 \mathrm{~nm}$. 


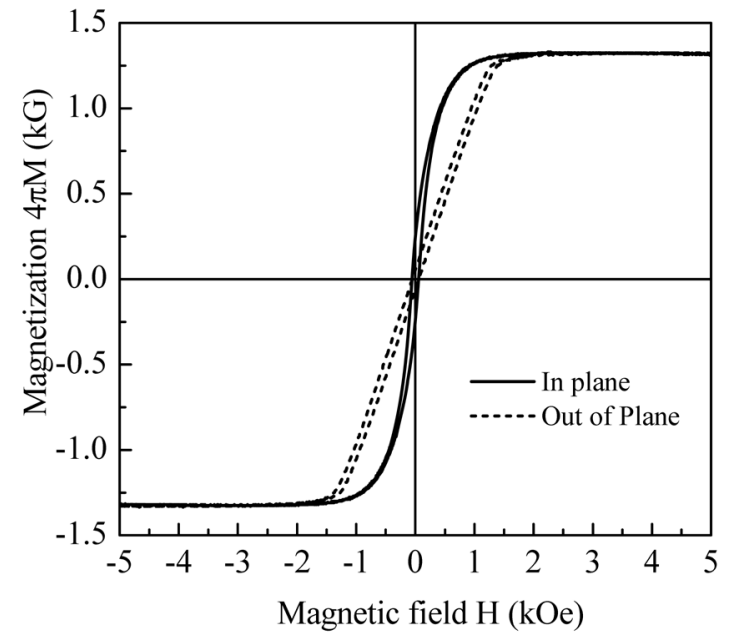

Fig. 3. Magnetization of the 1D-MPC.

field for out-of-plane was $1.5 \mathrm{kOe}$, which corresponds to Faraday configuration.

Fig. 4 shows transmittance spectrum of the fabricated 1D-MPC having high order PBGs, which there are the first $P B G$ in wavelength from 2200 to $2900 \mathrm{~nm}$, the second PBG from 820 to $930 \mathrm{~nm}$, the third PBG around $550 \mathrm{~nm}$ and the localized mode at $894 \mathrm{~nm}$ in the second PBG. Since film thicknesses in the 1D-MPC were different from the designed thicknesses as described before, wavelength of the localized mode in the second PBG was shifted from $900 \mathrm{~nm}$ to $894 \mathrm{~nm}$, and the center of the second PBG was also shifted from $900 \mathrm{~nm}$ to $860 \mathrm{~nm}$. Furthermore, as seen in Fig. 4, the narrow third PBG appeared around $550 \mathrm{~nm}$. However, the transmittance near the edge of the third PBG around $400 \mathrm{~nm}$ decreased drastically. This is the reason that the light was absorbed in the Bi:YIG defect layer of the 1D-MPC below $600 \mathrm{~nm}$, which can be understand by transmittance of single B:YIG film as shown in Fig. 5(a).

The Bi:YIG is well known to be a magnetic material with high transparency (Fig. 5(a)); maximum Faraday
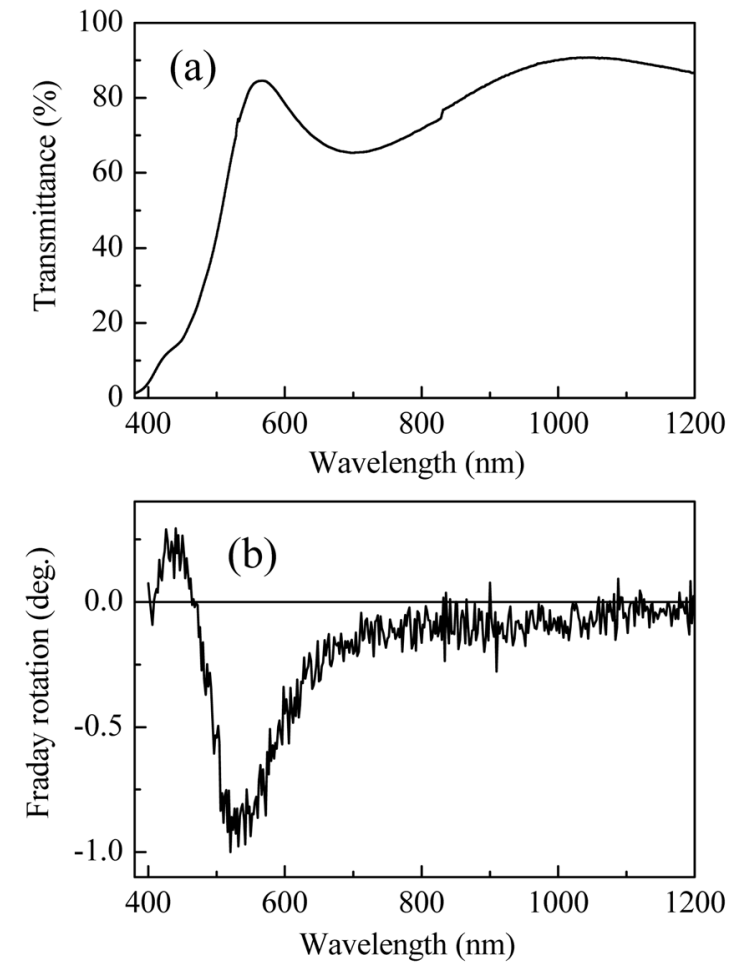

Fig. 5. (a) Transmittance and (b) Faraday rotation spectra of single Bi:YIG thin film with thickness of $250 \mathrm{~nm}$.

rotation occurs in the vicinity of $520 \mathrm{~nm}$ (Fig. 5(b)). Using the Bi:YIG as the defect layer of the 1D-MPC, enhancement of the Faraday rotation was obtained at the localized mode in the first PBG [1-3]. In this measurement of Faraday rotation of the 1D-MPC, magnetic field of $3 \mathrm{kOe}$ was applied, which is larger than the saturation magnetic filed of $1.5 \mathrm{kOe}$ (Fig. 3). Fig. 6 shows experimental and calculated spectra of (a) transmittance and (b) Faraday rotation of the 1D-MPC including the second PBG and the third PBG; the calculated spectra were obtained by using film thickness measured by FE-SEM. As seen in Fig. 6, at the localized mode of $900 \mathrm{~nm}$ in the second PBG, transmittance of $57 \%$ was obtained (Fig.

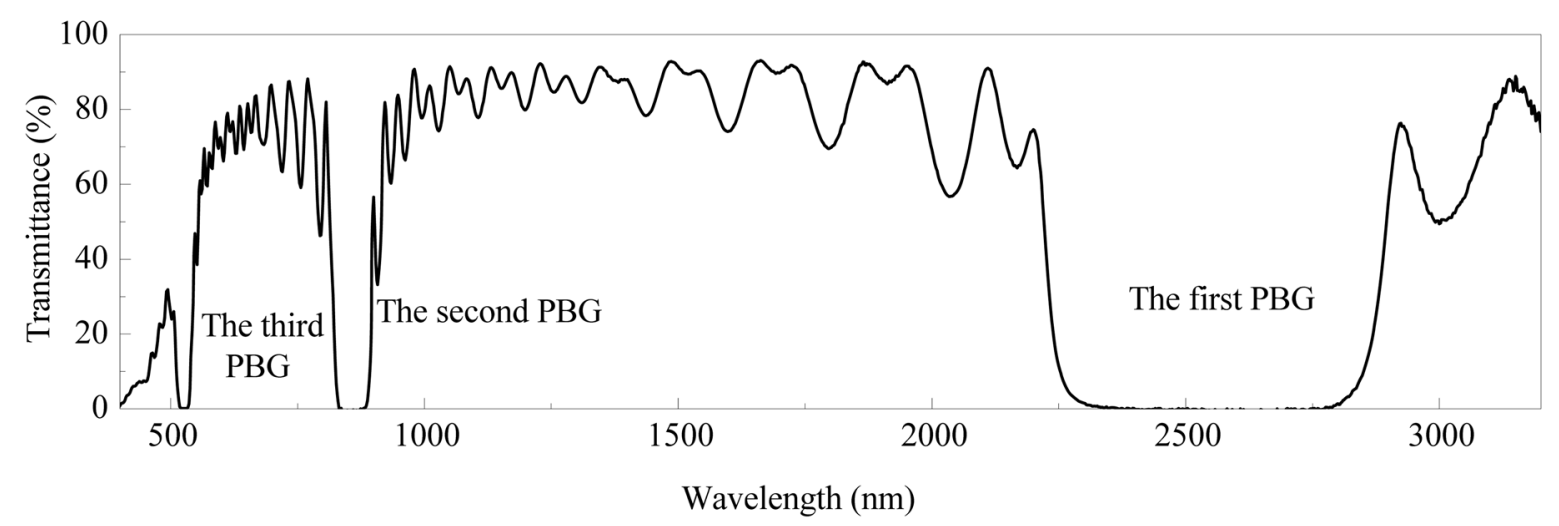

Fig. 4. Transmittance spectrum of the fabricated 1D-MPC having higher order PBGs. Three PBGs exist in the wavelength region. 

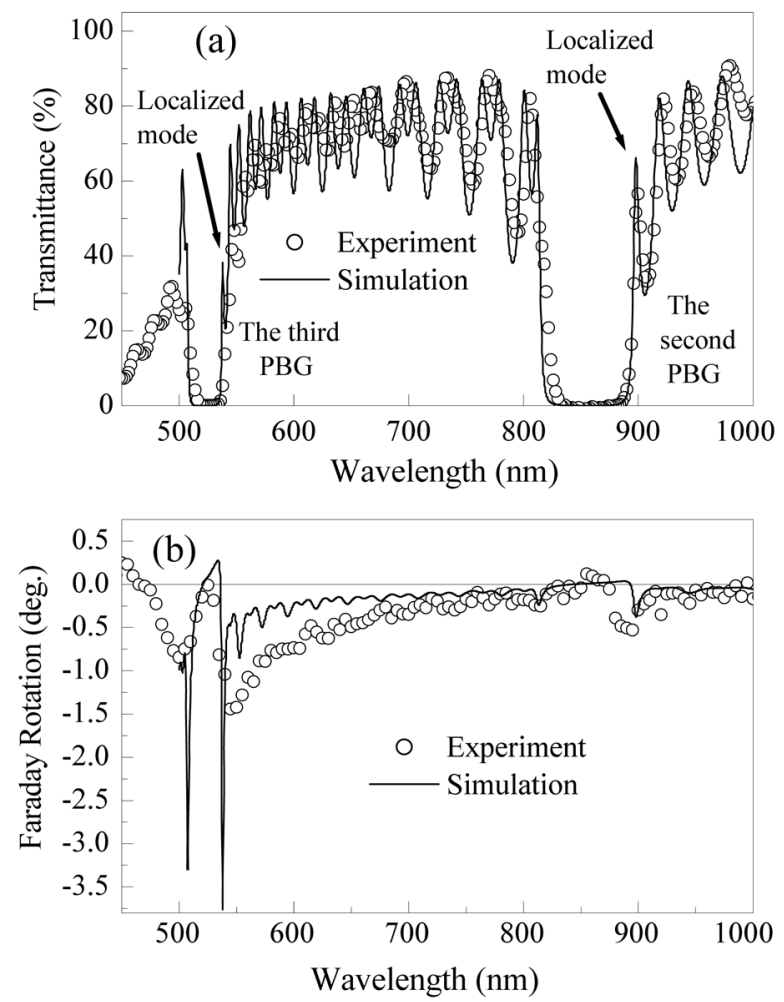

Fig. 6. Magnified spectra for (a) transmittance and (b) Faraday rotation of the 1D-MPC around the second and the third PBGs. Experimental data is denoted by open circle and calculated data is denoted by solid line.

6(a)), and enhancement of Faraday rotation angle of -0.5 degree was observed (Fig. 6(b)). In addition, at a localized mode in the third PBG vicinity of $540 \mathrm{~nm}$, transmittance of about $30 \%$ and Faraday rotation angle of -1.5 degree were obtained. These angles are larger than the Faraday rotation of -0.2 degree $(900 \mathrm{~nm})$ and -0.8 degree $(540$ $\mathrm{nm})$ for the single Bi:YIG film. Faraday rotation could be enhanced by increment of effective optical thickness of Bi:YIG, which was induced by reflection effect of light in periodic structure of the 1D-MPC. Feature of experimental spectra of transmittance and Faraday rotation (indicated by open circles) as shown in Fig. 6 is in good agreement with calculated spectra (indicated by solid lines). However, Faraday rotation angles in experiment and calculation were not so large as we expected before. It can be caused by shift of localized mode from center of the second PBG, therefore, accurate periodic structure is of importance for large Faraday rotation in the 1D-MPC

Faraday rotation angle of the 1D-MPC utilizing the second PBG was not so large in comparison with the 1DMPC utilizing the first PBG as described in ref. [1-3]. In the 1D-MPC having the localized mode at $900 \mathrm{~nm}$ in the second PBG, thickness of Bi:YIG defect layer is same to one of the 1D-MPC having the localized mode of the first PBG at $900 \mathrm{~nm}$, however, thickness of dielectric layers of $3 \lambda / 4$ for the second PBG-MPC is thicker than one of $\lambda / 4$ for the first PBG-MPC. Because extinction coefficient of $\mathrm{Ta}_{2} \mathrm{O}_{5}$ is not small as described in ref [2], absorption of light is large in the second PBG-MPC with thick $\mathrm{Ta}_{2} \mathrm{O}_{5}$ layers. Therefore, reflection effect and light localization in the Bi:YIG defect layer of the second PBG-MPC would be smaller than one of the first PBG-MPC. If we use a dielectric material with small extinction coefficient, large Faraday rotation might be obtained at the localized mode in the second PGB. Otherwise, thin $\mathrm{Ta}_{2} \mathrm{O}_{5}$ layers in periodic structure may be effective. This consideration can be generally applied to the MPC with Bragg refractors and defect layer.

\section{Conclusion}

We investigated the 1D-MPC utilizing high order photonic band gap. The localized modes appeared in the second and the third PBGs; enhancement of the Faraday rotation was observed at each localized mode. Optical and magneto-optical properties of their PBGs were simulated, which were in good agreement with experimentally obtained spectra. Comparing with the single Bi:YIG film, enhancement of Faraday rotation at the localized modes was observed. The guideline for obtaining larger Faraday rotation in the magnetophotonic crystals was examined.

\section{Acknowledgements}

This work was supported by Giant-in-Aid (S) (No. 17106004.) from the Ministry of Education, Culture, Sport and Technology of Japan.

\section{References}

[1] M. Inoue, K. Arai, T. Fujii, and M. Abe, J. Appl. Phys. 85, 5768 (1999).

[2] H. Kato, T. Matsushita, A. Takayama, M. Egawa, K. Nishimura, and M. Inoue, J. Appl. Phys. 93, 3906 (2003).

[3] H. Kato, T. Matsushita, A. Takayama, M. Egawa, H. Uchida, K. Nishimura, and M. Inoue, Trans. Magn. Soc. Japan, 4, 286-289 (2004).

[4] T. V. Murzina, R. V. Kapra, T. V. Dolgova, A. A. Fedyanin, O. A. Aktsipetrov, K. Nshimura, H. Uchida, and M. Inoue, Phys. Rev. B 70, 012407 (2004).

[5] A. A. Fedyanin, O. A. Aktsipetrov, D. Kobayashi, K. Nishimura, H. Uchida, and M. Inoue, IEEE Trans. Mag. 40, 2850 (2004). 